\title{
Serotype Distribution and Characteristics of the Minimum Inhibitory Concentrations of Streptococcus pneumoniae Isolated from Pediatric Patients in Kunming, China
}

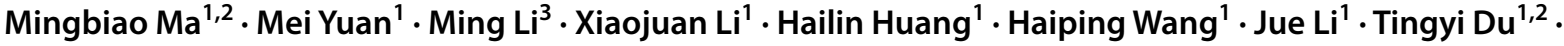 \\ Rongwei Huang ${ }^{4}$
}

Received: 30 August 2019 / Accepted: 6 November 2019 / Published online: 18 February 2021

(c) The Author(s) 2021

\begin{abstract}
Streptococcus pneumoniae (S. pneumoniae) is the main conditional pathogen of acute respiratory infection in infants, children, and older adults worldwide. It was great significant to identify the epidemic characteristics of serotypes and antibiotic susceptibility for the prevention and treatment of $S$. pneumoniae diseases. This research assessed the serotype distribution and the minimum inhibitory concentrations (MICs) of $S$. pneumoniae isolated from pediatric patients to provide information on the epidemiology and antibiotic resistance of $S$. pneumoniae in Kunming, China. A total of 140 S. pneumoniae isolates were collected from pediatric patients at Kunming Children's Hospital from January 2016 to October 2017. Serotype identification was done by Quellung reaction and multiplex polymerase chain reaction. MICs were determined by $E$-test. 140 isolates distributed in 13 types of serotypes. The top-three prevalent serotypes were 19F, 19A, and 6B. The immunization coverage rate of 13-valent pneumococcal conjugate vaccine (PCV) was relatively higher and should be introduced into the vaccination program in the region. $\mathrm{MIC}_{50}$ of penicillin, ceftriaxone, and levofloxacin was $1 \mu \mathrm{g} / \mathrm{mL}$. $\mathrm{MIC}_{50}$ for meropenem and vancomycin was $0.38 \mu \mathrm{g} / \mathrm{mL}$. $\mathrm{MIC}_{90}$ of penicillin, ceftriaxone, and levofloxacin was $1.5 \mu \mathrm{g} / \mathrm{mL}$ and that of meropenem and vancomycin was $0.5 \mu \mathrm{g} / \mathrm{mL}$. The $\mathrm{MIC}_{90}$ of erythromycin was $>256 \mu \mathrm{g} / \mathrm{mL}$. In summary, S. pneumoniae had low resistance rates to penicillin, ceftriaxone, levofloxacin, vancomycin, and meropenem, and these antibiotics could be the first-line agents for children with pneumococcal infections in Kunming.
\end{abstract}

\section{Introduction}

Streptococcus pneumoniae (S. pneumoniae) is a Grampositive bacteria. It is the most common pathogenic bacterial species that causes community-acquired infection in

Mingbiao Ma and Mei Yuan contributed equally to the study.

Tingyi Du

dutingyi@etyy.cn

Rongwei Huang

huangrongwei@etyy.cn

Mingbiao Ma

15096187255@163.com

Mei Yuan

815079752@qq.com

Ming Li

liming@etyy.cn

Xiaojuan Li

1708305337@qq.com

Hailin Huang

2262384809@qq.com
Haiping Wang 187000233@qq.com

Jue Li

997285158@qq.com

1 Department of Clinical Laboratory, Kunming Children's Hospital, Kunming 650500, China

2 Yunnan Key Laboratory of Children's Major Disease Research, Kunming 650500, China

3 Department of Respiratory Medicine, Kunming Children's Hospital, Kunming 650500, China

4 Department of Respiratory and Critical Care, Kunming Children's Hospital, Kunming 650500, China 
children, and can cause meningitis, otitis media, sinusitis, bacteremia, and pneumonia. It can even endanger life in severe cases [1]. According to a World Health Organization (WHO) report, there were about 700,000 to $1,000,000$ children worldwide die from pneumococcal infections every year, and most are children under 5 years of age $[2,3]$. The population of China is the largest in the world, and about 350,000 children aged $<5$ years died from pneumococcal infections each year from 2010 to 2015 [4]. This figure accounted for $10 \%$ of children who died worldwide from pneumococcal infections. Therefore, pneumococcal infections in children have become an important public-health issue all over the world and aroused the great attention of medical circles.

At the microscope level, there is a double-spherical arrangement of capsules, which are the main virulence factors of S. pneumoniae. Depending on the structure and composition of the capsular polysaccharide, S. pneumoniae can be divided into 46 serogroups and 90 serotypes at present. About 20 serotypes are associated with common $S$. pneumoniae-related diseases [5].

However, to reduce the disease burden of pneumococcal infections, two types of multivalent vaccines, a pneumococcal polysaccharide vaccine (PPV) and pneumococcal conjugate vaccines (PCVs), have been developed and proven to be an effective method for preventing pneumococcal infections worldwide. The 23-valent pneumococcal polysaccharide vaccine (PPV23) can target 23 pneumococcal serotypes (1, 2, 3, 4, 5, 6B, 7F, 8, 9N, 9V, 10A, 11A, 12F, 14, 15B, 17F, 18C, 19A, 19F, 20, 22F, 23F, and 33F). Three types of PCVs are in use. 7-valent pneumococcal conjugate vaccine (PCV7) can target seven pneumococcal serotypes $(4,6 \mathrm{~B}, 9 \mathrm{~V}, 14$, 18C, 19F, and 23F). PCV11 contains additional serotypes $1,3,5$, and 7F, and PCV13 includes extra 6A and 19A. PPV23 has a higher coverage rate of serotypes compared with any PCVs, but whether it produces protective antibodies is dependent upon stimulating B cells. PPV23 cannot produce a strong immune protective effect for infants and children aged $<2$ years. PCVs can activate $\mathrm{T}$ cells to provide sufficient immunologic help to produce antibodies and to stimulate immunological memory. PCVs are recommended for infants and young children with relatively immature B cells.

Currently, PPV23 and PCV7 are available commercially in China. However, several studies have found that serotypes are distributed differently in different periods, geographic areas, and populations [6]. Whether existing multivalent pneumococcal vaccines are suitable for children is dependent upon the serotype distribution of clinical pneumococcal isolates in a particular region.

Antibiotics are first-line treatment against pneumococcal infections, especially $\beta$-1Lactam antibiotics such as penicillin and ceftriaxone. However, with the widespread use of antibiotics in recent years, S. pneumoniae has become resistant to various antimicrobial drugs. In particular, the emergence of penicillin-resistant S. pneumoniae (PRSP) has become a big challenge for clinical treatment [7, 8].

Kunming, the capital city of Yunnan Province, is an important gateway to South Asia. This Chinese city has a population of seven million people. However, the development of economy and health care here has lagged behind that of other cities in China. Most of epidemiologic data of $S$. pneumoniae mainly focused on several developed cities including Beijing, Shanghai, and Guangzhou. The children here are rarely vaccinated with pneumococcal vaccines. Epidemiology and drug-resistance information for S. pneumoniae have been not yet available for Yunnan province.

We investigated, for the first time, the essential information about serotypes distribution and antibiotic resistance of S. pneumoniae in Kunming. This information is very important for S. pneumoniae infections in children in this region, especially for the introduction and policies of vaccination.

\section{Materials and Methods}

\section{Collection of Bacterial Isolates}

From January 2016 to October 2017, a total of 140 S. pneumoniae isolates were collected from pediatric patients at the Kunming Children's Hospital. These bacterial isolates were obtained from sputum, blood, eye secretions, broncho-alveolar lavage fluid (BALF), and middle-ear fluid. We inoculated the specimens onto agar plates containing 5\% sheep red blood cells (Autobio Diagnostics, Zhengzhou, China). We cultured them in an atmosphere of $5 \% \mathrm{CO}_{2}$ for $18-24 \mathrm{~h}$ at $37{ }^{\circ} \mathrm{C}$. We found the suspected colonies to be concave and accompanied by $\alpha$-hemolysis. Further confirmation was achieved by the presence of diplococci on Gram stain under the microscope views, test (optochin sensitivity, the bile susceptibility), and by use of an automatic bacterial identification system (VITEK2-compact, bioMerieux, Marcy-l'Étoile, France). These isolates were stored at $-80^{\circ} \mathrm{C}$ in frozen tubes containing equine serum (Microbank ${ }^{\mathrm{TM}}$, Pro Lab Diagnostics, Richmond Hill, ON, USA).

\section{Detection of Serotypes}

The serotypes/groups of all 140 strains were identified first with a Pneumotest-Latex kit (Statens Serum Institut, Copenhagen, Denmark) comprising 14 latex reagents pools (A to I and $\mathrm{P}$ to $\mathrm{T}$ ). The kit could identify the 23 vaccine serotypes at the type/group level by all 14 pools using the "chessboard" identification system. Type-specific antisera were used for full serotypes of serogroups 19, 6, and 15. Strains that could not be serotyped by the Quellung reaction were 
finally confirmed using multiplex polymerase chain reaction (PCR) targeting the cps locus using primers designed previously [9]. PCR was done in a 50 $\mu \mathrm{L}$ PCR mixture containing $25 \mu \mathrm{L}$ of $2 \times$ Multiplex PCR Buffer, $0.5 \mu \mathrm{L}$ of $0.2 \mu \mathrm{M}$ of each primer, $0.25 \mu \mathrm{L}$ of Multiplex PCR Enzyme Mix, $19.75 \mu \mathrm{L}$ of $\mathrm{dH}_{2} \mathrm{O}$, and $1 \mu \mathrm{L}$ of DNA template extracted from isolates (TaKaRa Biotechnology, Shiga, Japan). PCR products were visualized by electrophoresis on $1 \%$ agarose gel. The immunization coverage rates of PCV7, PCV13, and PPV23 were estimated by calculating the percentages of strains targeted by each vaccine in all of those isolates.

\section{Antimicrobial Susceptibility Test}

E-test and Mueller Hinton agar plates containing 5\% red blood cells from sheep (Autobio Diagnostics) were used to assess the minimum inhibitory concentrations (MICs) of 140 S. pneumoniae isolates for susceptibility to penicillin, ceftriaxone, meropenem, levofloxacin, vancomycin, and erythromycin. The results of the susceptibility testing to antibiotics were determined according to the guidelines provided by the Clinical and Laboratory Standards Institute (CLSI) 2018 [10]. For the susceptibility breakpoint of penicillin, $\mathrm{MIC} \leq 2 \mu \mathrm{g} / \mathrm{mL}$ was defined as "susceptible," $4 \mu \mathrm{g} / \mathrm{mL}$ was "intermediate," and MIC $\geq 8 \mu \mathrm{g} / \mathrm{mL}$ was "resistant." In addition, ATCC49619 was used as the quality-control strain to ensure the accuracy of results in each test. $\mathrm{MIC}_{50}$ and $\mathrm{MIC}_{90}$ represented the antibiotic concentrations at which $50 \%$ and $90 \%$ of the bacterial isolates could be inhibited, respectively.

\section{Statistical Analysis}

The data of the antimicrobial susceptibility were analyzed using WHONET 5.6 software (WHO, Geneva, Switzerland).

\section{Results}

\section{Information of Pediatric Patients and Isolates}

Streptococcus pneumoniae isolates were collected from 140 patients aged from 4 months to 9 years. The proportion of boys accounted for $58.57 \%(82 / 140)$ and that of girls was $41.43 \%$ (58/140). Sputum was the most common specimen source, accounting for $94.29 \%$ of samples (132 isolates), followed by eye secretions (4 isolates), middle-ear fluid (2 isolates), blood (1 isolate), and BALF (1 isolate).

\section{Serotype Distribution and Immunization Coverage Rates of Pneumococcal Vaccines}

Of all 140 isolates, 139 isolates were identified and distributed in 13 types of serotypes. One isolate could not be identified. The serotype distribution of all 140 S. pneumoniae isolates in different age groups is shown in Table 1 . In children aged $<2$ years and children aged $\geq 2$ years, the most prevalent serotype was $19 \mathrm{~F}$ ( $28.0 \%$ and $39.2 \%$, respectively), followed by $19 \mathrm{~A}(22.4 \%$ and $17.6 \%$, respectively) and $6 \mathrm{~B}$ (14.6\% and $13.7 \%$, respectively). Rare serotypes such as 6D, $7 \mathrm{C}$, and $10 \mathrm{~A}$ appeared mainly in children aged $<2$ years.

Of all 140 isolates, the immunization coverage rate of PCV7 and PCV13 was $54.29 \%$ (76/140) and $80.71 \%$ (114/140), respectively. In addition, the immunization coverage rate of PCV7 and PCV13 in children aged $<2$ years (51.69\% and $78.65 \%$, respectively) was lower compared that that of children aged $\geq 2$ years $(58.82 \%$ and $84.31 \%$, respectively). The immunization coverage rate of PPV23 in children aged $\geq 2$ years was higher than that of any PCV (Fig. 1).

\section{Antimicrobial Susceptibility Test}

The $\mathrm{MIC}_{50}$ and $\mathrm{MIC}_{90}$ of the $140 \mathrm{~S}$. pneumoniae isolates against to six common antibiotics are shown in Table 2. All results of ATCC49619 were within the reference range. The $\mathrm{MIC}_{50}$ of meropenem and vancomycin was $0.38 \mu \mathrm{g} / \mathrm{mL}$ and that of penicillin, ceftriaxone, and levofloxacin was $1 \mu \mathrm{g} /$ $\mathrm{mL}$. The $\mathrm{MIC}_{90}$ of penicillin, ceftriaxone, and levofloxacin was $1.5 \mu \mathrm{g} / \mathrm{mL}$, and that of meropenem and vancomycin was $0.5 \mu \mathrm{g} / \mathrm{mL}$. The $\mathrm{MIC}_{50}$ and $\mathrm{MIC}_{90}$ values of erythromycin were $>256 \mu \mathrm{g} / \mathrm{mL}$. The distributions of MICs for the $140 \mathrm{~S}$. pneumoniae isolates against to six common antibiotics are shown in Fig. 2. According to the CLSI 2018, no isolate resistant to penicillin, ceftriaxone, levofloxacin, or vancomycin was found. The resistance rate of 140

Table 1 Serotype distribution of 140 S. pneumoniae isolates in different age groups

\begin{tabular}{llll}
\hline Serotype & $\begin{array}{l}\text { All children } \\
n(\%)\end{array}$ & $\begin{array}{l}\text { Children } \\
\text { aged }<2 \text { years } \\
n(\%)\end{array}$ & $\begin{array}{l}\text { Children } \\
\text { aged } \geq 2 \text { years } \\
n(\%)\end{array}$ \\
\hline 19F & $45(32.1 \%)$ & $25(28.0 \%)$ & $20(39.2 \%)$ \\
19A & $29(20.7 \%)$ & $20(22.4 \%)$ & $9(17.6 \%)$ \\
6B & $20(14.2 \%)$ & $13(14.6 \%)$ & $7(13.7 \%)$ \\
15B/C & $13(9.2 \%)$ & $7(7.8 \%)$ & $6(11.7 \%)$ \\
$6 \mathrm{~A}$ & $8(5.7 \%)$ & $4(4.4 \%)$ & $4(7.8 \%)$ \\
23F & $8(5.7 \%)$ & $5(5.6 \%)$ & $3(5.8 \%)$ \\
15A & $6(4.2 \%)$ & $5(5.6 \%)$ & $1(1.9 \%)$ \\
14 & $3(2.1 \%)$ & $3(3.3 \%)$ & 0 \\
6C & $3(2.1 \%)$ & $3(3.3 \%)$ & 0 \\
$6 \mathrm{D}$ & $1(0.7 \%)$ & 0 & $1(1.9 \%)$ \\
7C & $1(0.7 \%)$ & $1(1.1 \%)$ & 0 \\
20 & $1(0.7 \%)$ & $1(1.1 \%)$ & 0 \\
10A & $1(0.7 \%)$ & $1(1.1 \%)$ & 0 \\
Untyped & $1(0.7 \%)$ & $1(1.1 \%)$ & 0 \\
Total & 140 & 89 & 51
\end{tabular}



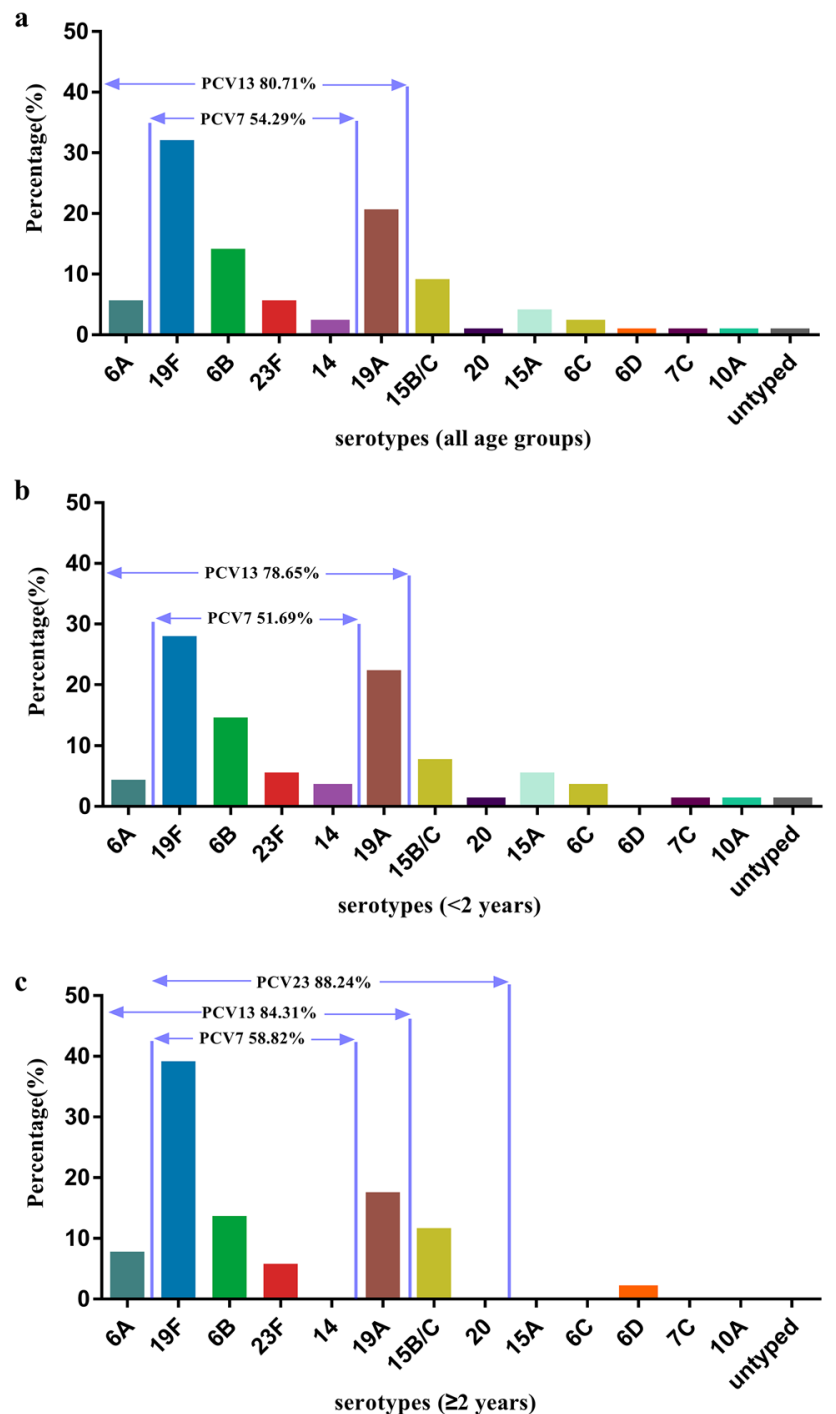

Fig. 1 Serotypes and immunization coverage rates of a PCV7 and PCV13 in 140 S. pneumoniae isolates, b PCV7 and PCV13 among children aged $<2$ years, and $\mathbf{c}$ PCV7, PCV13, and PPV23 among children aged $\geq 2$ years

Table 2 The $\mathrm{MIC}_{50}$ and $\mathrm{MIC}_{90}$ of $140 \mathrm{~S}$. pneumoniae isolates and distribution of MICs of isolates and ATCC49619 against to six common antibiotics

\begin{tabular}{lllll}
\hline Antibiotic & $\begin{array}{l}\mathrm{MIC}_{50} \\
(\mu \mathrm{g} / \mathrm{mL})\end{array}$ & $\begin{array}{l}\mathrm{MIC}_{90} \\
(\mu \mathrm{g} / \mathrm{mL})\end{array}$ & $\begin{array}{l}\text { MIC range of } \\
\text { isolates } \\
(\mu \mathrm{g} / \mathrm{mL})\end{array}$ & $\begin{array}{l}\text { MIC range of } \\
\text { ATCC49619 } \\
(\mu \mathrm{g} / \mathrm{mL})\end{array}$ \\
\hline Penicillin & 1 & 1.5 & $0.094-2$ & $0.25-0.38$ \\
Ceftriaxone & 1 & 1.5 & $0.008-3$ & $0.032-0.064$ \\
Meropenem & 0.38 & 0.5 & $0.008-1.5$ & $0.032-0.064$ \\
Levofloxacin & 1 & 1.5 & $0.38-4$ & 0.5 \\
Vancomycin & 0.38 & 0.5 & $0.032-0.75$ & $0.19-0.38$ \\
Erythromycin & $>256$ & $>256$ & $2->256$ & 0.094 \\
\hline
\end{tabular}

isolates to meropenem and erythromycin was 5\% and $100 \%$, respectively.

Furthermore, we analyzed the MICs of predominant serotypes (19F, 19A, and 6B) against penicillin. The distribution of MICs (in $\mu \mathrm{g} / \mathrm{mL}$ ) of serotypes 19F, 19A, and 6B was concentrated at 1.5, 1.0, and 0.75 , respectively (Fig. 3). At this time, we calculated the $\mathrm{MIC}_{50}$ and $\mathrm{MIC}_{90}$ of penicillin and found that the $\mathrm{MIC}_{50}$ and $\mathrm{MIC}_{90}$ of serotype $19 \mathrm{~A}$ were 1 and $1.5 \mu \mathrm{g} / \mathrm{mL}$, respectively, which was similar to the results of the $140 \mathrm{~S}$. pneumoniae isolates. However, the $\mathrm{MIC}_{50}$ of serotypes $19 \mathrm{~F}$ and $6 \mathrm{~B}$ for penicillin both were $0.75 \mu \mathrm{g} / \mathrm{mL}$ and less than that of the $140 \mathrm{~S}$. pneumoniae isolates.

\section{Discussion}

Streptococcus pneumoniae is the main conditional pathogen of acute respiratory infection in infants, children, and senior citizens worldwide. It also can cause large-scale epidemics and seriously endanger human health under certain environment [11]. In recent years, the resistance rates of $S$. pneumoniae have been increasing year by year, which have been related to the application of antibiotics in some areas. Hausdorff and colleagues revealed significant differences in the serotypes and resistance rates of $S$. pneumoniae in different regions [6]. Here, we showed, for the first time, the serotype distribution and the MICs of S. pneumoniae to provide information on the epidemiology and antibiotic resistance of $S$. pneumoniae in Kunming.

Identification of the epidemic characteristics of serotypes is extremely important for the prevention and treatment of S. pneumoniae-related diseases. To understand the serotype distribution of $S$. pneumoniae, surveillance has been carried out in various regions and countries. One systematic evaluation showed that the most common serotypes worldwide to be 1, 5, 6A, 6B, 14, 19F, and 23F [12]. In Canada, the most common serotypes were 19A, 7F, 3, and 22F. Serotypes 19A, 5, 7F, and 3 were predominant in Spain [13]. However, the dominant serotypes in China are 19F, 19A, 23F, 14, and 6B [14]. Hence, the serotypes of $S$. pneumoniae may vary with ethnicity and region. From the serotype distribution of 140 S. pneumoniae isolates in the present study, 14 serotypes of $S$. pneumoniae were carried by pediatric patients in Kunming. The distribution was relatively concentrated, mainly serotypes 19F, 19A, and 6B. These data are in accordance with the main epidemic serotypes of children documented in Shanghai, Suzhou, and Chongqing [15-17] but different for those from Beijing (19F, 14, 23F, 6B, and 19A) and Chengdu (19F, 6, 14, 23F, and 15B/C) [18, 19]. Besides, Shen and colleagues found that 19F, 6A/B, 19A, and 23 were frequent serotypes in Zunyi (a city near to Kunming) [20]. Such results may suggest that the difference in serotype distribution between areas close to each other is not obvious. 

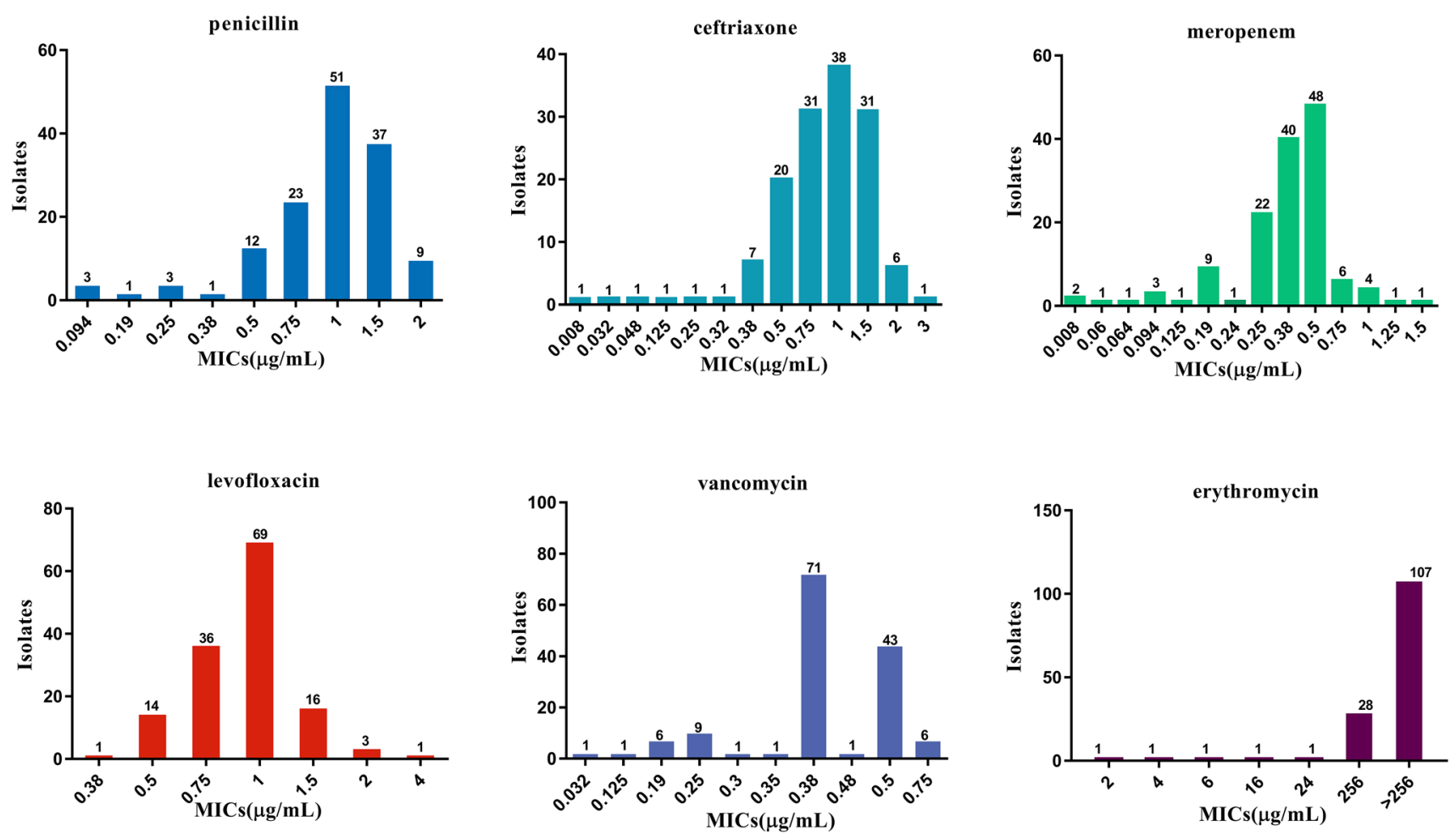

Fig. 2 Distribution of MICs of 140 S. pneumoniae isolates against six common antibiotics

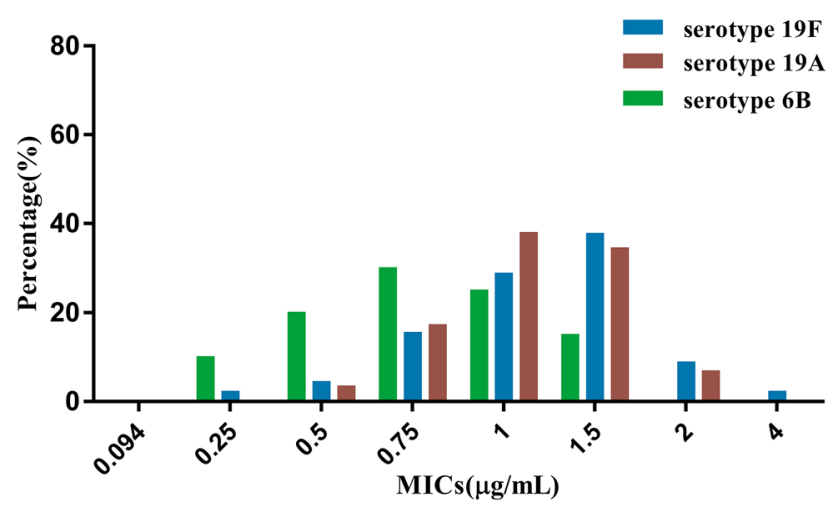

Fig. 3 Distribution of MICs of serotypes19F, 19A and 6B against penicillin

Serotyping of S. pneumoniae and evaluating the application of pneumococcal vaccines are important steps to prevent and treat pneumococcal infections and to understand their epidemic characteristics. PCV7 and PPV23 have been approved for marketing in China recently. Infants aged 3 to 23 months and children aged 24 to 59 months not vaccinated with PCV7 are recommended to be vaccinated with PCV7, whereas children $\geq 2$ years of age and adults can be vaccinated with PPV23. We showed that the immunization rate of PCV7 in children aged $<2$ years and that of PPV23 in children aged $\geq 2$ years was $51.69 \%$ and $88.24 \%$, respectively.
These data suggested that promotion of vaccination using PCV7 and PPV23 had preventive importance in China.

However, with the gradual promotion of a vaccine, some non-vaccine serotypes can increase in the proportion of isolates ("serotype replacement") [21]. In the present study, the increase in non-vaccine serotype 19A was $20.7 \%$. Because of targeting of serotype 19A, the immunization coverage rate of PCV13 increased and accounted for $80.71 \%$. Based on the serotype distribution of $S$. pneumoniae in our study, we believe that PCV13 should be introduced into the vaccination program in Kunming, and it may protect a greater proportion of the population from pneumococcal infections.

The resistance rate of penicillin in China is slightly lower than that in neighboring countries and regions, and the antibiotic resistance rates of $S$. pneumoniae among children vary greatly in different regions [22]. According to the results of CHINET in 2017, the resistance rates of penicillin, ceftriaxone, levofloxacin, vancomycin, and erythromycin in children and newborns were $1.6 \%, 13.2 \%$, $0.7 \%, 0 \%$ and $96.8 \%$, respectively, across China [23]. We showed that the resistance rates of penicillin, ceftriaxone, and levofloxacin in Kunming were lower than that in China. Six isolates were resistant to meropenem. The resistance rate of meropenem was lower than that reported by Jing et al. [17]. The reasons for this difference may be (i) because we used $E$-tests for drug- susceptibility testing, which is more likely to avoid false-positive resistance 
values than that using routine clinical methods and (ii) related to the region where is accustomed to use the ceftriaxone or meropenem rather than penicillin to treat pneumococcal infections.

Since the first discovery of PRSP in Australia in 1967, the resistance of $S$. pneumoniae to antibiotics such as $\beta$-1Lactams, macrolides, and tetracycline has been reported widely, and the resistance rate of penicillin is related to the geographic distribution [8, 24, 25]. Although S. pneumoniae has 90 serotypes, the serotype distribution of PRSP is relatively concentrated. In the United States, $96 \%$ of penicillinresistant serotypes of $S$. pneumoniae are $6 \mathrm{~B}, 14,23 \mathrm{~F}, 9 \mathrm{~V}$, 19A, and 19F. In Canada, 23F, 19A, 6B, and 9V are the main serotypes resistant to penicillin, and $14.82 \%$ of PRSP belong to serotypes 23F, 19A, 14, 6B, and 9V in France [6]. However, we did not find a PRSP isolate, which may be because penicillin is not often used as the first-line agent against pneumococcal infections in Kunming.

Ascertaining the distribution of MICs of antibiotics is significant conducive to surveying the changing trends of resistance rates in an area and guiding clinical antibiotic use. In our study, the distribution of MICs of penicillin was mainly $0.5-2 \mu \mathrm{g} / \mathrm{mL}$, and that of $\mathrm{MIC}_{50}$ and $\mathrm{MIC}_{90}$ was $1 \mu \mathrm{g} /$ $\mathrm{mL}$ and $1.5 \mu \mathrm{g} / \mathrm{mL}$, respectively. These data indicate that the sensitivity of penicillin to $S$. pneumoniae in Kunming was high, and that penicillin could be used for treatment of $S$. pneumoniae infections in Kunming. However, the characteristics of MICs in different serotypes showed differences. The distributions of MICs of serotypes 19F, 19A, and 6B was concentrated at $1.5,1$, and $0.75 \mu \mathrm{g} / \mathrm{mL}$, respectively, in the present study. The $\mathrm{MIC}_{50}$ in serotypes $19 \mathrm{~F}$ and $6 \mathrm{~B}$ was $0.75 \mu \mathrm{g} / \mathrm{mL}$ and was $<1 \mu \mathrm{g} / \mathrm{mL}$ in 19A. Although this change had no significant effect on qualitative determination of penicillin in our study, further investigation of the distribution of MICs of serotypes is necessary for long-term monitoring.

\section{Conclusions}

We found that 19F, 19A, and 6B were the most common serotypes of $S$. pneumoniae isolated from pediatric patients in Kunming. PCV13 was more useful than PCV7 in the region to prevent the spread of pneumococcal infections. $S$. pneumoniae had low resistance rates to penicillin, ceftriaxone, levofloxacin, vancomycin, and meropenem, and these antibiotics could be the first-line treatment for children with pneumococcal infections in this region. Moreover, larger sample size and longer monitoring should also be included in future studies to confirm our results and provide more detailed information on the epidemiology and antibiotic resistance of S. pneumoniae for the region.
Acknowledgements This project was supported by Grants from the Science and Technology Foundation of Yunnan Province of China (2016FB136), Science and Technology Foundation of Kunming City of China (2015-1-S-00564) and Health Science and Technology Personnel Training Foundation of Kunming City of China [2016-sw-20,2020-SW (reserve personnel)-109].

Author Contributions MM, MY, and TD conceived and designed experiments. XL, HH, and HW undertook antibiotic-susceptibility testing and serotyping. ML and RH contributed to collection of strains and case data. MM, MY, and TD wrote the manuscript. All authors read and approved the final version of manuscript.

\section{Compliance with Ethical Standards}

Conflict of interest The authors declare that they have no conflict of interest.

Ethical Approval The study protocol was approved by the Ethics Committee of Kunming Children's Hospital (Kunming, China).

Informed Consent Written informed consent was obtained from each participant's parents or legal guardians.

Open Access This article is licensed under a Creative Commons Attribution 4.0 International License, which permits use, sharing, adaptation, distribution and reproduction in any medium or format, as long as you give appropriate credit to the original author(s) and the source, provide a link to the Creative Commons licence, and indicate if changes were made. The images or other third party material in this article are included in the article's Creative Commons licence, unless indicated otherwise in a credit line to the material. If material is not included in the article's Creative Commons licence and your intended use is not permitted by statutory regulation or exceeds the permitted use, you will need to obtain permission directly from the copyright holder. To view a copy of this licence, visit http://creativecommons.org/licenses/by/4.0/.

\section{References}

1. Shibl AM (2008) Distribution of serotypes and antibiotic resistance of invasive pneumococcal disease isolates among children aged 5 years and under in Saudi Arabia (2000-2004). Clin Microbiol Infect 14(9):876-879. https://doi.org/10.111 $1 / j .1469-0691.2008 .02058 . x$

2. World Health Organization (2007) Pneumococcal conjugate vaccine for childhood immunization-WHO position paper. Wkly Epidemiol Rec 82(12):93-104

3. O'Brien KL, Wolfson LJ, Watt JP et al (2009) Burden of disease caused by Streptococcus pneumoniae in children younger than 5 years: global estimates. Lancet 374(9693):893-902. https://doi. org/10.1016/S0140-6736(09)61204-6

4. Zhou X, Liu J, Zhang Z et al (2016) Molecular characteristics of penicillin-binding protein $2 \mathrm{~b}, 2 \mathrm{x}$ and $1 \mathrm{a}$ sequences in Streptococcus pneumoniae isolates causing invasive diseases among children in Northeast China. Eur J Clin Microbiol Infect Dis 35(4):633635. https://doi.org/10.1007/s10096-016-2582-3

5. Kay EJ, Yates LE, Terra VS et al (2016) Recombinant expression of Streptococcus pneumoniae capsular polysaccharides in Escherichia coli. Open Bioinform J 6(4):150243. https://doi.org/10.1098/ rsob. 150243 
6. Hausdorff WP, Feikin DR, Klugman KP (2005) Epidemiological differences among pneumococcal serotypes. Lancet Infect Dis 5(2):83-93. https://doi.org/10.1098/rsob.150243

7. Zhao C, Sun H, Wang H et al (2012) Antimicrobial resistance trends among 5608 clinical gram-positive isolates in China: results from the gram-positive cocci resistance surveillance program (2005-2010). Diagn Microbiol Infect Dis 73(2):174-181. https://doi.org/10.1016/j.diagmicrobio.2012.03.003

8. Hansman D, Bullen MM (1967) A resistant pneumococcus. Lancet 290(7509):264-265. https://doi.org/10.1016/S0140 -6736(67)92346-X

9. Pai R, Gertz RE, Beall B (2006) Sequential multiplex PCR approach for determining capsular serotypes of Streptococcus pneumoniae isolates. J Clin Microbiol 44(1):124-131. https:// doi.org/10.1128/JCM.44.1.124-131.2006

10. Clinical and Laboratory Standards Institute (2018) Methods for dilution antimicrobial susceptibility tests for bacteria that grow aerobically; approved standard-ninth edition. Document M100. CLSI, Wayne

11. Lynch JP, Zhanel GG (2010) Streptococcus pneumoniae: epidemiology and risk factors, evolution of antimicrobial resistance, and impact of vaccines. Curr Opin Pulmon Med 16(3):217-225. https://doi.org/10.1097/MCP.0b013e3283385653

12. Johnson HL, Maria DK, Levine OS et al (2010) Systematic evaluation of serotypes causing invasive pneumococcal disease among children under five: the pneumococcal global serotype project. PLoS Med 7(10):e1000348. https://doi.org/10.1371/journ al.pmed. 1000348

13. Demczuk WHB, Martin I, Desai S et al (2013) Serotype distribution of invasive Streptococcus pneumoniae in adults 65 years of age and over after the introduction of childhood 13-valent pneumococcal conjugate vaccination programs in Canada, 20102016. Can J Microbiol 59(12):778-788. https://doi.org/10.1139/ cjm-2013-0614

14. Chen K, Zhang X, Shan W et al (2018) Serotype distribution of Streptococcus pneumoniae and potential impact of pneumococcal conjugate vaccines in China: a systematic review and metaanalysis. Hum Vaccin Immunother 14(7):1453-1463. https://doi. org/10.1080/21645515.2018.1435224

15. Yang F, Xu XG, Yang MJ et al (2008) Antimicrobial susceptibility and molecular epidemiology of Streptococcus pneumoniae isolated from Shanghai, China. Int J Antimicrob Agents 32(5):386391. https://doi.org/10.1016/j.ijantimicag.2008.05.004

16. Geng Q, Zhang T, Ding Y et al (2014) Molecular characterization and antimicrobial susceptibility of Streptococcus pneumoniae isolated from children hospitalized with respiratory infections in Suzhou, China. PLoS ONE 9(4):e93752. https://doi.org/10.1371/ journal.pone.0093752

17. Jing CM, Wang S (2016) Resistance and serotype of Streptococcus pneumoniae isolates from children in Chongqing from 2009 to 2014. Chin J Antibiot 41(1):64-69

18. Zhou L, Yu SJ, Gao W et al (2011) Serotype distribution and antibiotic resistance of 140 pneumococcal isolates from pediatric patients with upper respiratory infections in Beijing, 2010. Vaccine 29(44):7704-7710. https://doi.org/10.1016/j.vacci ne.2011.07.137

19. Xiao YH, Wang J, Zhu Y et al (2010) Resistance and serotype of 123 strains of Streptococcus pneumoniae. Chin J Vacc Immun 20(16):2377-2383

20. Shen M, Yao R, Yue H et al (2017) Serotype prevalence and antibiotic susceptibility patterns of pneumococcal isolates in Zunyi city, China. Saudi Med J 38(12):1243-1249. https://doi. org/10.15537/smj.2017.12.21090

21. Pelton SI, Huot H, Finkelstein JA et al (2007) Emergence of 19A as virulent and multidrug resistant pneumococcus in Massachusetts following universal immunization of infants with pneumococcal conjugate vaccine. Pediatr Infect Dis J 26(6):468-472. https://doi.org/10.1097/INF.0b013e31803df9ca

22. Zhao C, Li Z, Zhang F et al (2017) Serotype distribution and antibiotic resistance of Streptococcus pneumoniae isolates from 17 Chinese cities from 2011 to 2016. BMC Infect Dis 17(1):804. https://doi.org/10.1186/s12879-017-2880-0

23. Li SG, Hu FP, Zhuo Y et al (2018) Surveillance of bacterial resistance in children and newborns across China from 2014 to 2017. Nat Med J Chin 98(47):3279-3287

24. Poll TVD, Opal SM (2009) Pathogenesis, treatment, and prevention of pneumococcal pneumonia. Lancet 374(9700):1543-1556. https://doi.org/10.1016/S0140-6736(09)61114-4

25. Li Y, Metcalf BJ, Chochua S et al (2016) (2016) Penicillin-binding protein transpeptidase signatures for tracking and predicting Î2-lactam resistance levels in Streptococcus pneumoniae. Mbio 7(3):e00756-e816. https://doi.org/10.1128/mBio.00756-16

Publisher's Note Springer Nature remains neutral with regard to jurisdictional claims in published maps and institutional affiliations. 